\title{
Individual risk calculator to predict lymph node metastases in patients with submucosal (T1b) esophageal adenocarcinoma: a multicenter cohort study
}

Authors

Annieke W. Gotink ${ }^{1,}{ }^{,}$, Steffi E. M. van de Ven ${ }^{1,{ }^{*}}{ }^{\oplus}$, Fiebo J. C. ten Kate ${ }^{2,3}$, Daan Nieboer ${ }^{4}$, Lucia Suzuki², Bas L. A. M. Weusten $^{5,6}$, Lodewijk A. A. Brosens ${ }^{7}$, Richard van Hillegersberg ${ }^{8}$, Lorenza Alvarez Herrero ${ }^{6}$, Cees A. Seldenrijk ${ }^{9}$, Alaa Alkhalaf ${ }^{10}$, Freek C. P. Moll ${ }^{3}$, Erik J. Schoon ${ }^{11}$, Ineke van Lijnschoten ${ }^{12}$, Thjon J. Tang ${ }^{13}$, Hans van der Valk ${ }^{14}$, Wouter B. Nagengast ${ }^{15}$, Gursah Kats-Ugurlu ${ }^{16}$, John T. M. Plukker ${ }^{17}$, Martin H. M. G. Houben ${ }^{18}$, Jaap S. van der Laan ${ }^{19}$, Roos E. Pouw $^{20}$, Jacques J. G. H. M. Bergman ${ }^{20}$, Sybren L. Meijer ${ }^{21} \odot$, Mark I. van Berge Henegouwen ${ }^{22}$, Bas P. L Wijnhoven ${ }^{23}$, Pieter Jan F. de Jonge ${ }^{1}$, Michael Doukas², Marco J. Bruno ${ }^{1}$, Katharina Biermann ${ }^{2,{ }^{* *}}$, Arjun D. Koch 1, **

Institutions

1 Department of Gastroenterology and Hepatology, Erasmus MC Cancer Institute, University Medical Center, Rotterdam, the Netherlands

2 Department of Pathology, Erasmus MC Cancer Institute, University Medical Center, Rotterdam, the Netherlands

3 Department of Pathology, Isala Clinics, Zwolle, the Netherlands

4 Department of Public Health, Erasmus University Medical Center, Rotterdam, the Netherlands

5 Department of Gastroenterology and Hepatology, University Medical Center Utrecht, Utrecht, the Netherlands

6 Department of Gastroenterology and Hepatology, St. Antonius Hospital, Nieuwegein, the Netherlands

7 Department of Pathology, University Medical Center Utrecht, Utrecht, the Netherlands

8 Department of Surgery, University Medical Center Utrecht, Utrecht, the Netherlands

9 Department of Pathology, Pathology DNA, St. Antonius Hospital, Nieuwegein, the Netherlands

10 Department of Gastroenterology and Hepatology, Isala Clinics, Zwolle, the Netherlands

11 Department of Gastroenterology and Hepatology, Catharina Hospital, Eindhoven, the Netherlands

12 Department of Pathology, PAMM, Eindhoven, the Netherlands

13 Department of Gastroenterology and Hepatology, ljsselland Hospital, Capelle aan den Ijssel, the Netherlands

14 Department of Pathology, Ijselland Hospital, Capelle aan den Ijssel, the Netherlands

15 Department of Gastroenterology and Hepatology, University Medical Center Groningen, Groningen, the Netherlands

16 Department of Pathology, University Medical Center Groningen, Groningen, the Netherlands
17 Department of Surgery, University Medical Center Groningen, Groningen, the Netherlands

18 Department of Gastroenterology and Hepatology, Haga Teaching Hospital, Den Haag, the Netherlands

19 Department of Pathology, Haga Teaching Hospital, Den Haag, the Netherlands

20 Department of Gastroenterology and Hepatology, Cancer Center Amsterdam, Amsterdam Gastroenterology Endocrinology Metabolism, Amsterdam University Medical Centers, Amsterdam, the Netherlands

21 Department of Pathology, Amsterdam University Medical Centers, Amsterdam, the Netherlands

22 Department of Surgery, Amsterdam University Medical Centers, Amsterdam, the Netherlands

23 Department of Surgery, Erasmus MC Cancer Institute, University Medical Center, Rotterdam, the Netherlands

submitted 13.7.2020

accepted after revision 24.2.2021

published online 24.2.2021

Bibliography

Endoscopy 2022; 54: 109-117

DOI 10.1055/a-1399-4989

ISSN 0013-726X

(C) 2021. Thieme. All rights reserved.

Georg Thieme Verlag KG, Rüdigerstraße 14,

70469 Stuttgart, Germany

丹 Supplementary material

Supplementary material is available under https://doi.org/10.1055/a-1399-4989

* Co-first authors

** Co-last authors 
Scan this QR-Code for the author commentary.

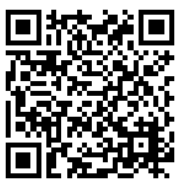

Corresponding author

Arjun D. Koch, MD, PhD, Department of Gastroenterology and Hepatology, Erasmus MC Cancer Institute, University Medical Center, Postbox 2040, 3000 CA, Rotterdam, The Netherlands a.d.koch@erasmusmc.nl

\section{ABSTRACT}

Background Lymph node metastasis (LNM) is possible after endoscopic resection of early esophageal adenocarcinoma (EAC). This study aimed to develop and internally validate a prediction model that estimates the individual risk of metastases in patients with pT1b EAC.

Methods A nationwide, retrospective, multicenter cohort study was conducted in patients with PT1b EAC treated with endoscopic resection and/or surgery between 1989 and 2016. The primary end point was presence of LNM in surgical resection specimens or detection of metastases during follow-up. All resection specimens were histologically reassessed by specialist gastrointestinal pathologists. Subdistribution hazard regression analysis was used to develop the prediction model. The discriminative ability of this model was assessed using the c-statistic.

Results 248 patients with pT1b EAC were included. Metastases were seen in 78 patients, and the 5 -year cumulative incidence was $30.9 \%$ (95\% confidence interval [CI] 25.1\%$36.8 \%$ ). The risk of metastases increased with submucosal invasion depth (subdistribution hazard ratio [SHR] 1.08, $95 \% \mathrm{Cl} 1.02-1.14$, for every increase of $500 \mu \mathrm{m}$ ), lymphovascular invasion (SHR 2.95, $95 \% \mathrm{Cl} 1.95-4.45$ ), and for larger tumors (SHR 1.23, 95\%Cl 1.10-1.37, for every increase of $10 \mathrm{~mm}$ ). The model demonstrated good discriminative ability (c-statistic $0.81,95 \% \mathrm{Cl} 0.75-0.86$ ).

Conclusions $A$ third of patients with pT1b EAC experienced metastases within 5 years. The probability of developing post-resection metastases was estimated with a personalized predicted risk score incorporating tumor invasion depth, tumor size, and lymphovascular invasion. This model requires external validation before implementation into clinical practice.

\section{Introduction}

Esophageal adenocarcinoma (EAC) is an aggressive cancer, carrying a 5-year survival rate of $19 \%$ [1]. The most important prognostic factor for survival is the presence of lymph node metastasis (LNM) [2]. Radical esophagectomy with lymphadenectomy has long been the standard treatment for EAC, including early cancer. Endoscopic resection is a safe and effective treatment and is indicated for the curative management of early EAC [3]. Additional surgery after endoscopic resection is advocated when the risk of LNM outweighs the risk of mortality associated with esophagectomy [4].

Histopathological characteristics, such as lymphovascular invasion (LVI), poor tumor differentiation, and invasion into the submucosa of more than $500 \mu \mathrm{m}$, are associated with a risk of LNM [5-7]. When EAC is limited to the esophageal mucosa (T1a), the risk of LNM is only $1 \%-2 \%$ and radical endoscopic resection of the lesion suffices [4, 8-11]. When EAC invades the submucosa (T1b), guidelines recommend additional surgical resection of the esophagus and locoregional lymph nodes [4, 9]. The prevalence of LNM in esophagectomy specimens varies between 0 and $78 \%$ for T1b EACs and depends on the extent of invasion into the submucosa [7,12-15]. Superficial submucosal invasion $(\mathrm{sm} 1: \leq 500 \mu \mathrm{m})$ was associated with variable LNM risk in previous studies (0-22\%), while deep submucosal invasion was associated with higher LNM risk, ranging from 26\%-36\% $(\mathrm{sm} 2: 501-1000 \mu \mathrm{m})$ to $50 \%-78 \%(\mathrm{sm} 3:>1000 \mu \mathrm{m})[12,15-$ 17].

In the challenging clinical scenario when LNM is not seen on imaging, management depends on metastasis risk assessment.
Not every patient with pT1b EAC develops LNM, while surgery is associated with morbidity, mortality, and decreased quality of life $[18,19]$. Endoscopic resection might be a good alternative for selected patients with PT1sm1 EAC in combination with "low risk" histopathological characteristics, such as well-tomoderate differentiation (G1/G2) and absent LVI [20]. Only $1.9 \%$ of these patients developed LNM [20]. Other studies also reported LNM risk of $<2 \%$ in patients with pT1sm 1 EAC with "low risk" histopathological characteristics [21-23]. In contrast, the LNM risk is reported to be higher (up to $9 \%$ ) in patients with pT1sm1 EAC with at least one "high risk" histopathological characteristic, such as poor tumor differentiation or LVI [24]. The European Society of Gastrointestinal Endoscopy guidelines state that $\mathrm{R} 0$ resection of pT1bsm 1, G1-2, LVI- is potentially curative and the decision about whether or not to perform additional treatment should be individualized in these patients in terms of morbidity and mortality risk vs LNM risk [25].

Although many studies have described separate histopathological risk factors associated with LNM, no appropriate clinical tool is available that incorporates all accepted prognostic histopathological parameters to accurately predict the LNM risk on an individual basis. Little is known about how the individual risk increases with the accumulation of more than one histopathological feature. The aim of this study was to develop and internally validate a prediction model based on histopathological variables that estimates the risk of LNM or distant metastases in individual patients with pT1b EAC. 


\section{Methods}

\section{Study design}

We performed a nationwide, retrospective, multicenter cohort study in collaboration with the Netherlands Cancer Registry (NCR). Since 1989, all patients diagnosed with pT1b EAC in the Netherlands have been included in the NCR. Patients diagnosed with PT1b EAC between 1989 and 2016 were selected for the current study. Eight hospitals participated in the study and were expert centers in EAC treatment. Patients with pT1b EAC were included if they were treated with primary surgery or endoscopic resection (with or without adjuvant surgery). Patients were excluded if they were treated with chemoradiotherapy prior to surgery, as we could not reliably assess whether LNM developed. We also excluded patients with no histopathology data available for review, when histological parameters could not be reassessed, or when no patient data were available in medical charts. The study was approved by the Medical Ethical Review Committee of the Erasmus Medical Center in Rotterdam, the Netherlands (MEC-2016-050) and by all local Medical Ethical Review Committees at the participating centers. Clinical data were collected from medical records, pathology reports, and endoscopy reports (see File $1 \mathbf{s}$ in the online-only Supplementary material).

\section{Histopathological reassessment}

Owing to renewed pathology insights and different classification systems over the years, all resection specimens were histologically reassessed for the following parameters: submucosal invasion depth $(\mu \mathrm{m})$, differentiation grade, and LVI. This was independently performed by three specialist gastrointestinal pathologists (F.tK., M.D., K.B.) for 84 patients, as explained in detail in our previous study [26]. The interobserver agreement was good for differentiation grade $(\mathrm{K}=0.77)$, excellent for $\mathrm{LVI}$ $(\mathrm{K}=0.88)$, and moderate for submucosal invasion depth $(\mathrm{K}=$ $0.60)$ [26]. For all other patients $(n=164)$, histopathological reassessment for differentiation grade and LVI was performed by one pathologist (F.tK) because the interobserver agreement was good and excellent, respectively, for these histopathological variables [26]. Submucosal invasion was assessed in a consensus meeting by two pathologists (K.B., M.D.) in the remaining 164 patients because the interobserver agreement was moderate for submucosal invasion depth [26]. The reassessment is explained in supplementary File $\mathbf{2} \mathbf{s}[27,28]$.

After histopathological reassessment, patients were excluded if the tumor was located in the cardia or when tumor location was not known. Patients with vertical R1 or Rx endoscopic resection with residual tumor in biopsy or with residual EAC in surgical resection specimen were also excluded. In these cases, we could not reliably assess invasion depth or LVI. Patients with vertical $\mathrm{R} 1$ or $\mathrm{Rx}$ after endoscopic resection were included if no residual tumor was found during surgery.

\section{End points}

The primary end point was the presence of LNM in surgically resected specimens ( $\geq 12$ resected lymph nodes), or the development of metastases during follow-up. Owing to the use of dif- ferent surgical resection techniques over time, with less extensive lymphadenectomy in the past, the development of metastases during follow-up was used as a surrogate end point for LNM in cases with fewer than 12 lymph nodes present. In cases of an endoscopic resection prior to surgical resection, the tumor was found in the endoscopic resection specimen and lymph node status in the surgical resection specimen. When no additional surgery was performed after endoscopic resection, the development of metastases during follow-up was used as a surrogate end point, and patients were excluded if biopsy-proven residual tumor was found after endoscopic resection.

\section{Follow-up}

Patient follow-up data were retrieved until November 2019. A minimum follow-up period of 2 years was required in cases with $<12$ lymph nodes resected during surgery or when no surgery was performed. Patients who died within 2 years after primary treatment with an unknown cause of death were excluded because it was not clear whether these patients died as a result of metastases; therefore only patients with a known cause of death were included because it was clear whether metastases had developed or not.

\section{Sample size calculation}

The prevalence of LNM in T1b EAC varies between 0 and $78 \%$ in previous studies [5-7, 12-15]. In these studies, LNM was found in $110 / 456$ patients $(24 \%$, $95 \%$ confidence interval $[\mathrm{Cl}] 20 \%-$ $28 \%$ ) who underwent esophagectomy. At least 10 events were needed per predictor in our model. We predefined that we would incorporate all four histopathological predictors: differentiation grade, submucosal invasion, LVI, and tumor size. A minimum of 40 patients with LNM were needed in our calculator. Using the lower $\mathrm{Cl}$ for the prevalence of LNM in T1b EAC ( $20 \%$ ), a sample size of at least 200 patients was needed for the study.

\section{Statistical analyses}

Baseline characteristics are described using standard descriptive statistics. Continuous data are presented as mean (range) for normally distributed data and median (interquartile range [IQR]) for skewed data. Categorical data are presented with frequencies and percentages.

\section{Univariable and multivariable analysis}

Different follow-up periods and the competing risk of all-cause/ non-EAC mortality were taken into account using the Fine and Gray model. In the Fine and Gray model, subdistribution hazard ratios (SHRs) are estimated, describing the effect of covariates on the subdistribution hazard. SHRs and associated $95 \% \mathrm{Cls}$ were calculated for candidate predictors. The $95 \% \mathrm{Cls}$ were calculated through bootstrapping.

Based on previous literature about prediction model development, all variables with a $P$ value of $<0.2$ at univariable analysis were incorporated into multivariable analysis [29]. The following variables were included in univariable analysis: sex, LVI, tumor differentiation grade, submucosal invasion depth, and 
tumor size. Microscopic or macroscopic tumor size was used for analysis.

Information about tumor size was missing in 38 of 248 patients (15.3\%). We assumed these data were missing at random and used multiple imputation to impute the missing values 10 times. Separate analyses were performed on the 10 imputed datasets and results were subsequently pooled using Rubin rules.

Statistical analyses were performed in R version 3.6.3., the mice package was used for multiple imputation, and the riskRegression package was used to fit the Fine and Gray method.

\section{Prediction model and discriminative ability}

Only variables that were statistically significant $(P<0.05)$ after multivariable analysis were included in the final prediction model. For completeness, nonsignificant variables were also incorporated into the prediction model to check whether they altered the predicted scores. Submucosal depth invasion was incorporated as a continuous variable (absolute depth invasion in $\mu \mathrm{m}$ ) in the prediction model and later divided into different sm classifications in the score chart. Tumor size was also incorporated as a continuous variable in the prediction model and divided into $<20 \mathrm{~mm}$ and $\geq 20 \mathrm{~mm}$ in the score chart; this cutoff value was chosen based on previous studies [5, 24].

The discriminative ability of the model was assessed using the Harrell's concordance statistic (c-statistic), which varies between 0.5 (noninformative model) and 1.0 (perfect model). The model was internally validated using bootstrapping to calculate the Harrell's concordance statistic and to limit the risk of overfitting [29]. The coefficients from the subdistribution hazard regression were used to calculate the probability of developing LNM within 5 years (regression formula is presented in supplementary File $\mathbf{3 s}$ ).

Analyses were carried out using SPSS version 25 (IBM Corp., Armonk, New York, USA) for descriptive statistics and survival analysis using Kaplan-Meier, and R programming language, version 3.3.6 (R Foundation for Statistical Computing, Vienna, Austria. URL https://www.R-project.org) for univariable and multivariable analysis, cumulative incidence, and prediction model. All tests were two-sided. For final multivariable analysis, a two-sided test with a $P$ value of $<0.05$ was considered statistically significant.

\section{Results}

\section{Study flow chart}

A total of 524 patients with T1b EAC were selected from the NCR. After medical chart review, 90 patients were excluded ( Fig.1). Histopathological reassessment was performed for 434 T1b EACs, and 115 patients were excluded because pathology did not reveal pT1b EAC $(n=76)$ or the tumor was not located in the esophagus $(n=39)$. Medical charts of the 319 remaining patients with pT1b EAC were reviewed again and another 71 patients were excluded ( $\triangleright$ Fig. 1). A total of 248 patients were included in the final analysis.

\section{NCR search [524 patients] Period: 1989-2016}

Exclusion [90 patients]

- Pathology material not available for revision [42]

- Too little pathology material available [6]

- No patient data available [36]

- Chemo-radiotherapy prior to surgery [6]

T1b EAC [434 patients] Pathology reassessment

Exclusion [115 patients]

- pT1a EAC [66]

- pT2 EAC [7]

- No malignancy [3]

- Tumor located in the cardia or missing tumor location [39]

pT1b EAC [319 patients]

Exclusion [71 patients]

- Missing follow-up information or inadequate follow-up [27]

- Metastases; primary tumor unknown (esophagus or colon) [1]

- pT1b R1 or Rx (vertical) endoscopic resection, with residual EAC in biopsy or surgical resection specimen [43]

Inclusion [248 patients]

$\mid$

No LNM or distant metastases [170]

LNM or distant metastases during surgery or follow-up [78]

Distant metastases [6]

- Detected during follow-up [6]

LNM [49]

- Detected during follow-up [2]

- Detected during surgery [47]

LNM and distant metastases [23]

- Both detected during follow-up [4]

- LNM detected during surgery, distant metastases detected during follow-up [19]

- Fig. 1 Flow chart of patients. NCR, Netherlands Cancer Registry; EAC, esophageal adenocarcinoma; LNM, lymph node metastases.

\section{Patient and tumor characteristics}

Baseline characteristics are presented in $>$ Table 1. The median age of the cohort was 65.6 years (IQR 57.8-72.5) and $87.5 \%$ were male. Most patients were treated with primary surgery (166/248; 66.9\%); additional surgery was performed in 49/82 
- Table 1 Clinicopathological features of the study cohort $(n=248)$.

\begin{tabular}{|l|l|}
\hline Sex, $\mathbf{n}(\%)$ & $217(87.5)$ \\
\hline - Male & $31(12.5)$ \\
\hline - Female & $65.6(57.8-72.5)$ \\
\hline Age, median (IQR), years & \\
\hline Tumor location, $\mathbf{n}(\%)$ & $7(2.8)$ \\
\hline - Mid-esophagus & $178(71.8)$ \\
\hline - Distal esophagus & $63(25.4)$ \\
\hline - Gastroesophageal junction & \\
\hline Primary management, $\mathbf{n}(\%)$ & $82(33.1)$ \\
\hline Endoscopic resection & 78 \\
\hline - EMR & 4 \\
\hline - ESD & $166(66.9)$ \\
\hline Surgery & 114 \\
\hline - Transhiatal & 5 \\
\hline - Laparoscopic transhiatal & 38 \\
\hline - Transthoracic & 4 \\
\hline - Thoraco laparoscopic & 5 \\
\hline - Missing & \\
\hline
\end{tabular}

Vertical endoscopic resection margins, $\mathbf{n}(\%)$ *

\begin{tabular}{|l|l|}
\hline . R0 & $59(72.0)$ \\
\hline " R1 & $17(20.7)$ \\
\hline " Rx & $6(7.3)$ \\
\hline
\end{tabular}

\begin{tabular}{|l|l|}
\hline Barrett's, $\mathbf{n}(\%)$ & \\
\hline " Yes & $173(69.8)$ \\
\hline " No & $36(14.5)$ \\
\hline " Missing & 39 \\
\hline
\end{tabular}

\section{Tumor size}

- Median (IQR), mm $24.0(15.0-32.0)$

- Missing, $\mathrm{n}$ 29

\section{Differentiation grade, $\mathbf{n}(\%)$}

- G1/2 $162(65.3)$

- $\mathrm{G} 3 / 4$ $86(34.7)$

Sm invasion, $\mathbf{n}(\%)$

\begin{tabular}{|l|l|}
\hline " Sm1 & $56(22.6)$ \\
\hline " Sm2 & $51(20.6)$ \\
\hline " Sm3 & $141(56.9)$ \\
\hline Submucosal invasion, median (IQR), $\mu \mathrm{m}$ & $1300(590-2495)$ \\
\hline Presence of LVI, $\mathbf{n}(\%)$ & \\
\hline " No & $196(79.0)$ \\
\hline " Yes & $52(21.0)$ \\
\hline
\end{tabular}

- Table 1 (Continuation)

\section{Sex, n (\%)}

Metastases, $n$ (\%)

\begin{tabular}{|l|l|}
\hline - No & $170(68.5)$ \\
\hline - LNM & $49(19.8)$ \\
\hline - Distant metastasis & $6(2.4)$ \\
\hline - LNM + distant metastasis & $23(9.3)$ \\
\hline
\end{tabular}

IQR, interquartile range; EMR, endoscopic mucosal resection; ESD, endoscopic submucosal dissection; sm, submucosal; LVI, lymphovascular invasion; LNM, lymph node metastases.

* Calculated for 82 patients in whom endoscopic resection was performed.

(59.8\%) patients who were treated with endoscopic resection. Of patients who were only treated by endoscopic resection $(n=$ 31), 30/31 (96.8\%) did not develop metastases during followup. Clinical N stage was cN0 in $180 / 248$ patients ( $72.6 \%$ ), cN1 in $42 / 248$ patients (16.9\%), and unknown before endoscopic resection in $17 / 248$ patients (6.9\%). The total number of patients with cN1 stage, including patients in whom imaging was performed after endoscopic resection, was 50 . These patients were treated with surgery, and 27/50 (54.0\%) were confirmed to have LNM after histopathological assessment.

Vertical resection margins were positive (R1) in pathology reports of $17 / 82$ patients (20.7\%) who underwent endoscopic resection. Additional surgical resection was performed in all patients and no residual tumor was found in surgical resection specimens.

Resection margins were reported as "unable to determine" $(\mathrm{Rx})$ in $6 / 82$ patients (7.3\%). Additional surgery was performed in all patients and no residual tumor was found in surgical resection specimens.

\section{Metastases}

The median follow-up time was 5.5 years (IQR 4.9-7.7) in patients treated with endoscopic resection only and when $<12$ lymph nodes were present in surgical resection specimens. The median follow-up time was 3.3 years (IQR 1.8-5.3) in patients treated with primary surgery with $\geq 12$ lymph node dissections during surgery. The 5-year cumulative incidence of metastases was $30.9 \%(95 \% \mathrm{Cl} 25.1 \%-36.8 \%)$ ( Fig. 2). In total, 78 patients developed metastases. In 6/78 patients, only distant metastases developed. All had undergone surgery (without LNM) for primary PT1b EAC. In only 2/6 patients were $>12$ lymph nodes (range 14-30) resected during surgery.

The majority of patients only developed LNM (49/78) and all these patients were treated with surgery; LNM was found during surgery in 47 patients. LNM was found during follow-up in only 2/49 patients; in one of these patients, adequate lymph nodes $(n=18)$ had been resected during surgery.

Both LNM and distant metastases were found in 23/78 patients. One patient was treated with endoscopic resection only (due to comorbidity), and both LNM and distant metastases were detected during follow-up. A total of 22 patients were 


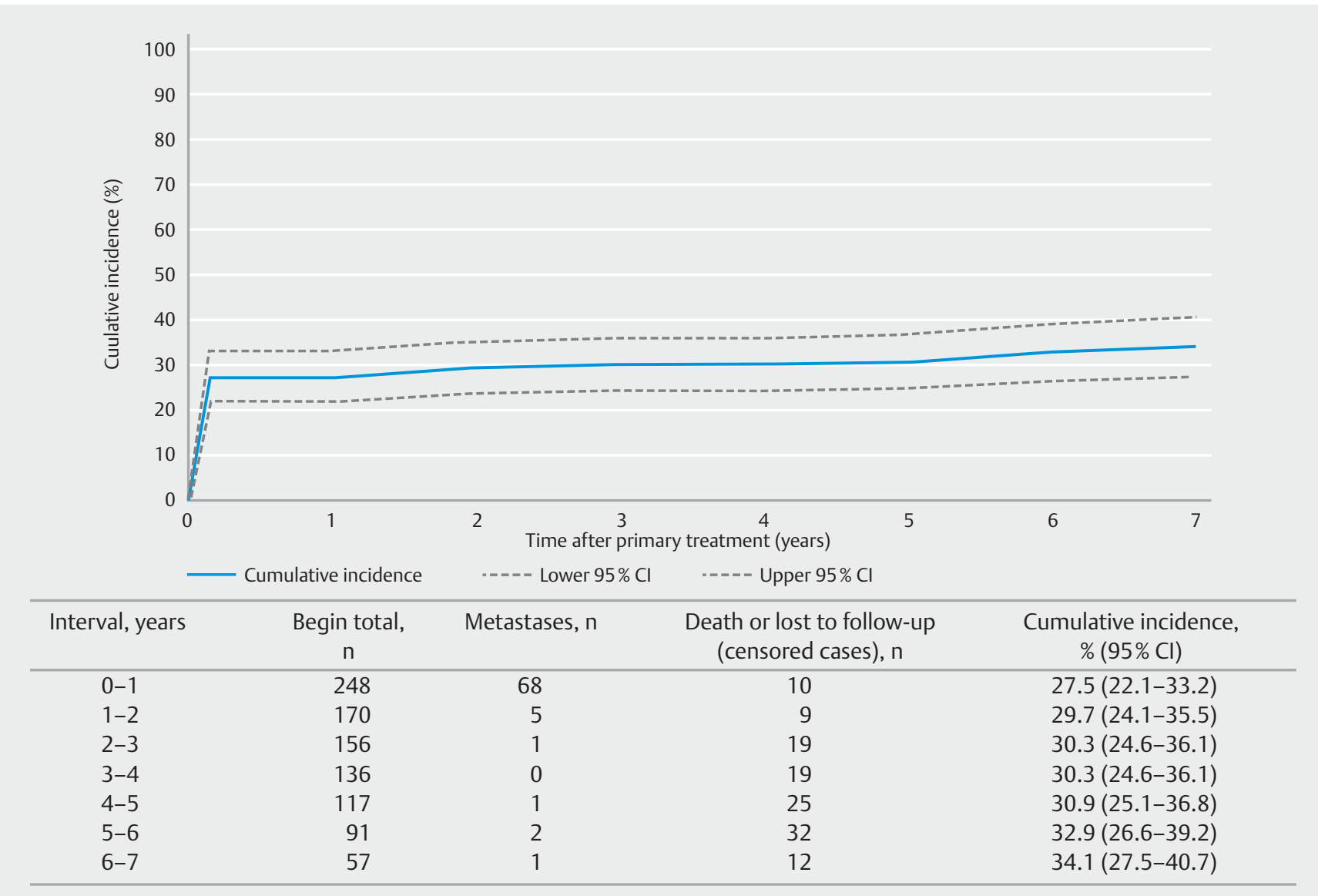

Fig. 2 Cumulative incidence of developing metastases, with death (not related to esophageal adenocarcinoma or metastases) as competing risk. EAC, esophageal adenocarcinoma; $\mathrm{Cl}$, confidence interval.

treated with surgery. LNM was found in the surgical resection specimen in 19/22 patients and during follow-up in 3/22 patients; in 2/3 patients, $>12$ lymph nodes had been resected during surgery. All distant metastases were detected during follow-up.

Of all detected LNMs $(n=72)$, the majority were found in the surgical resection specimen $(66 / 72 ; 91.7 \%)$. For all patients who developed metastases (LNM or distant metastases) during follow-up $(n=31)$, the median time to detection was 1.9 years (IQR 1.2-4.9). The disease-specific 5-year survival rate after primary treatment was $87.5 \%$ (Fig. 4s).

\section{Prediction model}

In multivariable analysis, the risk of developing metastases was higher for LVI-positive tumors (SHR 2.95, $95 \% \mathrm{CI} 1.95-4.45$ ), for tumors with deeper submucosal invasion (SHR 1.08, $95 \% \mathrm{Cl}$ 1.02-1.14), and for larger tumors (SHR 1.23, $95 \% \mathrm{Cl} 1.10-1.37$ ) ( $\triangleright$ Table 2). For every increase in invasion depth of $500 \mu \mathrm{m}$, the subdistribution hazard of developing metastases increased by 1.1. For every increase in tumor size of $10 \mathrm{~mm}$, the subdistribution hazard of developing metastases increased by 1.2. These variables were all incorporated into the prediction model. Incorporating differentiation grade into the prediction model did not alter the outcomes and therefore this variable was not incorporated into the score chart ( $\triangleright$ Fig. 3 ).

The risk of developing metastases for different combinations of histological variables is presented in the score chart ( $\mathbf{F i g . 3}$ ). The 5-year risk of developing metastases ranged from 5.9\% (95\% $\mathrm{Cl} 2.3-11.2$ ) for patients with pT1, sm 1, LVI- tumors $<20 \mathrm{~mm}$ to $70.1 \%$ (95\%Cl 60.5-78.7) for patients with pT1,sm3,LVI+tumors $\geq 20 \mathrm{~mm}$. After internal validation using bootstrapping, the prediction model demonstrated good discriminative ability with a c-statistic of $0.81(95 \% \mathrm{Cl} 0.75-0.86)$.

\section{Discussion}

The selection of patients who need additional treatment due to the risk of LNM after endoscopic resection of pT1b EAC can be challenging. Not all T1b EACs are alike when it comes to metastasis risk; however, they are regarded as such in most guidelines when it comes to make rigorous treatment decisions. Although individual risk factors associated with metastases have been described, little is known about how these risk factors interrelate or whether combining them may improve estimation of LNM risk. A clinical tool that incorporates accepted prognostic parameters for LNM risk on an individual basis is not yet available [5-7]. We established a prediction model that includes his- 
- Table2 Univariable and multivariable subdistributional hazard regression analysis of risk factors associated with metastases (no metastases $n=170$, metastases $n=78$ ).

\begin{tabular}{|c|c|c|c|c|}
\hline \multirow[t]{2}{*}{ Variable } & \multicolumn{2}{|c|}{$\begin{array}{l}\text { Univariable subdistributional hazard } \\
\text { regression analysis }\end{array}$} & \multicolumn{2}{|c|}{$\begin{array}{l}\text { Multivariable subdistributional hazard } \\
\text { regression analysis }\end{array}$} \\
\hline & SHR $(95 \% \mathrm{Cl})$ & $P$ value & SHR (95\%Cl) & $P$ value \\
\hline Sex, n (\%) & & & - & - \\
\hline - Female & Reference & & & \\
\hline - Male & $1.51(0.74-3.10)$ & 0.26 & & \\
\hline \multicolumn{5}{|l|}{ Differentiation grade } \\
\hline - G1/2 (good/moderate) & Reference & & Reference & \\
\hline - G3/4 (poor/undifferentiated) & $1.78(1.20-2.65)$ & $<0.01$ & $1.01(0.66-1.55)$ & 0.96 \\
\hline Submucosal invasion (per $500 \mu \mathrm{m}$ ) & $1.13(1.08-1.18)$ & $<0.01$ & $1.08(1.02-1.14)^{1}$ & $<0.01$ \\
\hline \multicolumn{5}{|l|}{ LVI } \\
\hline - No & Reference & & Reference & \\
\hline - Yes & $3.58(2.45-5.22)$ & $<0.01$ & $2.95(1.95-4.45)$ & $<0.01$ \\
\hline Tumor size (per 10 mm) & $1.39(1.25-1.53)$ & $<0.01$ & $1.23(1.10-1.37)^{2}$ & $<0.01$ \\
\hline
\end{tabular}

\begin{tabular}{l|l|l|l}
\hline & LVI-, \% (95\%Cl) & LVI+, \% (95\%Cl) & Tumor size \\
\hline sm1 & $5.9(2.3-11.2)$ & $15.7(6.0-29.3)$ & \\
\hline sm2 & $7.3(2.6-13.8)$ & $19.3(6.3-36.8)$ & $<20 \mathrm{~mm}$ \\
\hline sm3 & $14.1(7.9-21.9)$ & $34.7(19.7-50.8)$ & \\
\hline sm1 & $16.1(6.2-29.2)$ & $38.8(17.0-61.4)$ & \\
\hline sm2 & $19.4(8.6-32.2)$ & $45.6(20.8-67.9)$ & $\geq 20 \mathrm{~mm}$ \\
\hline sm3 & $35.2(25.8-44.7)$ & $70.1(60.5-78.7)$ & \\
\hline
\end{tabular}

Fig. 3 Score chart for 5-year metastases risk (both lymph node metastases and distant metastases) for different combinations of histopathological variables in patients with PT1b esophageal adenocarcinoma. LVI, lymphovascular invasion; Cl, confidence interval; sm, submucosa.

topathological tumor characteristics and estimates the individual risk of metastases in patients with pT1b EAC. This tool can be used in shared decision making about whether or not to undergo adjuvant treatment after minimally invasive endoscopic resection.

Patients treated with endoscopic resection or surgery for pT1b EAC were analyzed as one cohort to prevent possible selection bias. Endoscopic mucosal resection was introduced in 2001, and a selection bias would therefore have been created when separating patients included after 2001 based on treatment modality (endoscopic resection or surgery), because patients with relatively favorable tumor characteristics might have been treated more often with endoscopic resection in- stead of surgery. The median follow-up time was 5.5 years in patients treated with endoscopic resection or when $<12$ lymph nodes were present in surgical resection specimens. Because of this long follow-up period, we believe that analyzing both groups as a single cohort is acceptable.

In our cohort, approximately one third of patients had metastases. Risk factors for developing metastases were the presence of LVI and increased submucosal invasion depth and tumor size. After internal validation, the prediction model demonstrated good accuracy (c-statistic of 0.81 ). Based on our model, the estimated risk of developing metastases within 5 years of endoscopic resection or surgery ranged between $5.9 \%$ and $70.1 \%$, depending on different combinations of histopathological variables. The high metastases risk for specific $\mathrm{pT} 1 \mathrm{~b}$ EACs is the direct result of the combination of all poor histopathological parameters. This risk can even exceed the risk of metastases for tumors with a higher T stage that do not present with LVI [30].

The 5-year cumulative incidence of developing metastases was $30.9 \%(95 \% \mathrm{Cl} 25.1 \%-36.8 \%)$ in our cohort, which is in line with reported LNM rates $(0-78 \%)$ [7, 12-15, 24, 31, 32]. Previous studies have shown that LVI, poor tumor differentiation, progressive invasion depth, and larger tumor size are associated with LNM $[5,7,13,32]$. We confirmed LVI, increasing invasion depth, and tumor size to be significant independent risk factors for metastases. In contrast, differentiation grade was only found to be significant in univariable analysis.

Studies have suggested a subgroup of patients with pT1b EAC in which conservative treatment after endoscopic resection may be safe $[5,23,24,32]$. Graham et al. suggested that patients with $\mathrm{pT} 1, \mathrm{G} 1 / 2, \mathrm{sm} 1, \mathrm{LVI}-, \mathrm{R} 0 \mathrm{EAC}$ can be treated with 
endoscopic resection alone because these patients $(n=13)$ did not develop LNM during follow-up [32]. In addition, this study suggested conservative treatment after endoscopic resection of pT1bsm1 EACs with one high-risk pathological feature because $23 \%$ of the conservatively treated high-risk tumors developed LNM during follow-up [32]. However, the number of included patients in this study was too small to make recommendations [32]. Another study reported LNM in only $2 \%$ of "lowrisk" pT1b EAC patients; endoscopic resection was therefore suggested as an alternative treatment to esophagectomy [24]. A prediction model was lacking in these studies.

A study in which a prediction model was developed based on a weighted scoring system, showed that tumor size and LVI were predictors for LNM in patients with T1 EAC (both T1a and $\mathrm{T} 1 \mathrm{~b})$, with a c-statistic of $0.82(95 \% \mathrm{Cl} 0.75-0.89)$ [13]. This study suggested that esophagectomy should be considered in patients with a high risk score (> 5 points). However, patients with T1a tumors were also included and LNM was only predicted directly after surgery instead of taking follow-up into account. In addition, T1b tumors were not classified according to depth of infiltration (sm1-3) [13].

In the current large, nationwide, multicenter cohort study, histopathological reassessment and classification of all pT1b EACs was performed by consensus agreement between gastrointestinal pathologists with great expertise in EAC. The incorporated histopathological tumor characteristics were therefore valid, without missing data. Included patients were analyzed as a single cohort, regardless of primary treatment. An adequate follow-up period was guaranteed in patients treated with endoscopic resection and in patients with $<12$ resected lymph nodes during surgery. This is the first study with an adequate sample size that provides a good estimate for metastatic risk in patients with pT1b EAC.

However, the results of our model should be interpreted with caution due to limitations of the study. First, the retrospective study design could have resulted in selection and information bias. Several patients were excluded because of missing data. In addition, there was no standardized follow-up regimen. We are convinced, however, that it is not feasible to perform a study of this magnitude in a prospective design on T1b EAC. A second limitation and possible source of heterogeneity is the combined results of endoscopic and surgical resection specimens. Different specimen handling, sampling, and processing of endoscopic and surgical resection specimens ( $2 \mathrm{~mm}$ vs. $5 \mathrm{~mm}$ ) may theoretically lead to an underestimation of the deepest invasion in surgical specimens and introduce bias in histological interpretation. We are aware that treatment methods have changed over time and different surgical resection techniques are used, with less extensive lymphadenectomy being performed in the past. This is, however, inevitable in retrospective studies. We therefore used the development of metastases during follow-up as a surrogate end point in these patients. A third limitation is that no additional immunohistochemical staining was performed for the assessment of LVI, because formalin-fixed paraffin-embedded tissue blocks were not readily available to us. Additional immunohistochemical staining in combination with hematoxylin and eosin staining may improve the detection of LVI [33]. The final limitation is that we did not perform external validation of the prediction model. We do not know whether our prediction model, which is mainly based on surgical data, can reliably be used to calculate LNM risk in patients treated with endoscopic resection. One can argue that the performance of the model cannot be simply transferred to patients treated with endoscopic resection. External validation of the prediction model, using histological outcomes diagnosed in endoscopic resection specimens of T1b EAC, is necessary to assess whether the model has adequate predictive value to be used in clinical practice, where the focus is on deciding whether or not patients treated with endoscopic resection for T1b EAC will benefit from additional surgery. This validation is desirable in order to test the model strength when implementing it into clinical practice. The current predicted individual metastases risk is an estimation based on a statistical model; these estimations do carry a degree of uncertainty.

In conclusion, one third of patients with pT1b EAC developed metastases. A personalized risk could be predicted based on the presence or absence of each histopathological characteristic, with good discriminative ability. Deep submucosal tumor invasion, the presence of LVI, and larger tumor size were poor prognostic factors for metastases.

\section{Competing interests}

A. D. Koch has received consultancy fee from ERBE Elektromedizin and Pentax Medical. He has received research support from Dr Falk Pharma. M. J. Bruno is a consultant for Boston Scientific, Cook Medical and Pentax Medical. He has received support for industry and investigator initiated studies from Boston Scientific, Cook Medical, Pentax Medical, Mylan, ChiRoStim and 3M. M. I van Berge Henegouwen is consultant for Mylan, Johnson \& Johnson, Alesi Surgical and Medtronic, and received research grants from Olympus and Stryker.

References

[1] Siegel RL, Miller KD, Jemal A. Cancer statistics, 2019. CA Cancer J Clin 2019; 69: 7-34

[2] van der Schaaf M, Johar A, Wijnhoven B et al. Extent of lymph node removal during esophageal cancer surgery and survival. J Natl Cancer Inst 2015; 107: djv043

[3] Chevaux JB, Piessevaux H, Jouret-Mourin A et al. Clinical outcome in patients treated with endoscopic submucosal dissection for superficial Barrett's neoplasia. Endoscopy 2015; 47: 103-112

[4] Fitzgerald RC, di Pietro M, Ragunath K et al. British Society of Gastroenterology guidelines on the diagnosis and management of Barrett's oesophagus. Gut 2014; 63: 7-42

[5] Leers JM, DeMeester SR, Oezcelik A et al. The prevalence of lymph node metastases in patients with $\mathrm{T} 1$ esophageal adenocarcinoma a retrospective review of esophagectomy specimens. Ann Surg 2011; 253: $271-278$

[6] Liu L, Hofstetter WL, Rashid A et al. Significance of the depth of tumor invasion and lymph node metastasis in superficially invasive (T1) esophageal adenocarcinoma. Am J Surg Pathol 2005; 29: 1079-1085

[7] Barbour AP, Jones M, Brown I et al. Risk stratification for early esophageal adenocarcinoma: analysis of lymphatic spread and prognostic factors. Ann Surg Oncol 2010; 17: 2494-2502 
[8] Dunbar KB, Spechler SJ. The risk of lymph-node metastases in patients with high-grade dysplasia or intramucosal carcinoma in Barrett's esophagus: a systematic review. Am J Gastroenterol 2012; 107: 850862

[9] Spechler S], Sharma P, Souza RF et al. American Gastroenterological Association technical review on the management of Barrett's esophagus. Gastroenterology 2011; 140: e18-52

[10] Pech O, May A, Manner $\mathrm{H}$ et al. Long-term efficacy and safety of endoscopic resection for patients with mucosal adenocarcinoma of the esophagus. Gastroenterology 2014; 146: 652-660

[11] Alvarez HerreroL, Pouw RE, van Vilsteren FG et al. Safety and efficacy of multiband mucosectomy in 1060 resections in Barrett's esophagus. Endoscopy 2011; 43: 177-183

[12] Sepesi B, Watson TJ, Zhou D et al. Are endoscopic therapies appropriate for superficial submucosal esophageal adenocarcinoma? An analysis of esophagectomy specimens J Am Coll Surg 2010; 210: 418427

[13] Lee L, Ronellenfitsch U, Hofstetter WL et al. Predicting lymph node metastases in early esophageal adenocarcinoma using a simple scoring system. J Am Coll Surg 2013; 217: 191-199

[14] Stein HJ, Feith M, Bruecher BL et al. Early esophageal cancer: pattern of lymphatic spread and prognostic factors for long-term survival after surgical resection. Ann Surg 2005; 242: 566-575

[15] Westerterp M, Koppert LB, Buskens C] et al. Outcome of surgical treatment for early adenocarcinoma of the esophagus or gastroesophageal junction. Virchows Arch 2005; 446: 497-504

[16] Kaneshiro DK, Post JC, Rybicki L et al. Clinical significance of the duplicated muscularis mucosae in Barrett esophagus-related superficial adenocarcinoma. Am J Surg Pathol 2011; 35: 697-700

[17] Bollschweiler E, Baldus SE, Schroder W et al. High rate of lymph-node metastasis in submucosal esophageal squamous-cell carcinomas and adenocarcinomas. Endoscopy 2006; 38: 149-156

[18] Linden PA, Towe CW, Watson T] et al. Mortality after esophagectomy: analysis of individual complications and their association with mortality. J Gastrointest Surg 2020; 24: 1948-1954

[19] Finks JF, Osborne NH, Birkmeyer JD. Trends in hospital volume and operative mortality for high-risk surgery. N Engl J Med 2011; 364: 2128-2137

[20] Manner H, Pech O, Heldmann Y et al. Efficacy, safety, and long-term results of endoscopic treatment for early stage adenocarcinoma of the esophagus with low-risk sm 1 invasion. Clin Gastroenterol Hepatol 2013; 11: 630-635
[21] Manner H, May A, Pech O et al. Early Barrett’s carcinoma with “lowrisk" submucosal invasion: long-term results of endoscopic resection with a curative intent. Am J Gastroenterol 2008; 103: 2589-2597

[22] Scholvinck D, Kunzli H, Meijer S et al. Management of patients with T1b esophageal adenocarcinoma: a retrospective cohort study on patient management and risk of metastatic disease. Surg Endosc 2016; 30: 4102-4113

[23] Alvarez HerreroL, Pouw RE, van Vilsteren FG et al. Risk of lymph node metastasis associated with deeper invasion by early adenocarcinoma of the esophagus and cardia: study based on endoscopic resection specimens. Endoscopy 2010; 42: 1030-1036

[24] Manner $\mathrm{H}$, Pech $\mathrm{O}$, Heldmann $\mathrm{Y}$ et al. The frequency of lymph node metastasis in early-stage adenocarcinoma of the esophagus with incipient submucosal invasion (pT1b sm1) depending on histological risk patterns. Surg Endosc 2015; 29: 1888-1896

[25] Pimentel-Nunes P, Dinis-Ribeiro M, Ponchon T et al. Endoscopic submucosal dissection: European Society of Gastrointestinal Endoscopy (ESGE) Guideline. Endoscopy 2015; 47: 829-854

[26] Gotink AW, ten Kate FJ, Doukas M et al. Do pathologists agree with each other on the histological assessment of PT1b oesophageal adenocarcinoma? United European Gastroenterol J 2019; 7: 261-269

[27] Bosman FT, Carneiro F, Hruban RH, Theise ND. WHO classification of tumours of the digestive system. 4th edn.3 Lyon: International Agency for Research on Cancer; 2010

[28] The Paris endoscopic classification of superficial neoplastic lesions. esophagus, stomach, and colon: November 30 to December 1, 2002. Gastrointest Endosc 2003; 58: S3-43

[29] Steyerberg EW. Clinical prediction models - a practical approach to development, validation, and updating. 2nd edn. New York: SpringerVerlag; 2009

[30] Siewert JR, Stein HJ, Feith $M$ et al. Histologic tumor type is an independent prognostic parameter in esophageal cancer: lessons from more than 1,000 consecutive resections at a single center in the Western world. Ann Surg 2001; 234: 360-367

[31] Buskens C], Westerterp M, Lagarde SM et al. Prediction of appropriateness of local endoscopic treatment for high-grade dysplasia and early adenocarcinoma by EUS and histopathologic features. Gastrointest Endosc 2004; 60: 703-710

[32] Graham D, Sever N, Magee C et al. Risk of lymph node metastases in patients with T1b oesophageal adenocarcinoma: a retrospective single centre experience. World J Gastroenterol 2018; 24: 4698-4707

[33] Hosono I, Miyahara R, Furukawa K et al. Use of immunostaining for the diagnosis of lymphovascular invasion in superficial Barrett's esophageal adenocarcinoma. BMC Gastroenterol 2020; 20: 175 